\title{
Renewed Global Partnerships and Redesigned Roadmaps for Rabies Prevention and Control
}

\author{
Tiziana Lembo, ${ }^{1,2}$ Michaël Attlan, ${ }^{3}$ Hervé Bourhy, ${ }^{4}$ Sarah Cleaveland, ${ }^{2}$ Peter Costa, ${ }^{1}$ Katinka \\ de Balogh, ${ }^{5}$ Betty Dodet, ${ }^{6}$ Anthony R. Fooks, ${ }^{7}$ Elly Hiby, ${ }^{8}$ Fernando Leanes, ${ }^{9}$ François-Xavier \\ Meslin, ${ }^{10}$ Mary Elizabeth Miranda, ${ }^{1}$ Thomas Müller, ${ }^{11}$ Louis H. Nel, ${ }^{12}$ Charles E. Rupprecht, ${ }^{13}$ \\ Noël Tordo, ${ }^{4}$ Abbigail Tumpey, ${ }^{13}$ Alexander Wandeler, ${ }^{14}$ and Deborah J. Briggs ${ }^{1}$ \\ ${ }^{1}$ Global Alliance for Rabies Control, 529 Humboldt Street, Suite 1, Manhattan, KS 66502, USA \\ ${ }^{2}$ Institute of Biodiversity, Animal Health, and Comparative Medicine, College of Medicine, Veterinary and Life Sciences, University of \\ Glasgow, Graham Kerr Building, Glasgow G12 8QQ, UK \\ 3 Sanofi Pasteur, 2 avenue Pont Pasteur, 69367 Lyon Cedex 07, France \\ ${ }^{4}$ Institut Pasteur, 25 rue du Docteur Roux, 75724 Paris Cedex 15, France \\ ${ }^{5}$ Food and Agriculture Organization of the United Nations, Viale delle Terme di Caracalla, 00153 Rome, Italy \\ ${ }^{6}$ Dodet Bioscience, 6 bis rue de Verdun, 69300 Caluire et Cuire, France \\ ${ }^{7}$ Animal Health and Veterinary Laboratories Agency, Woodham Lane, New Haw, Addlestone, Surrey KT15 3NB, UK \\ ${ }^{8}$ World Society for the Protection of Animals, Programmes Department, 222 Grays Inn Road, London WC1X 8HB, UK \\ ${ }^{9}$ Pan American Health Organization, World Health Organization, PANAFTOSA, Avenue Presidente Kennedy 7778, 25040-004 Duque \\ de Caxias CEP, RJ, Brazil \\ ${ }^{10}$ World Health Organization, 20 Avenue Appia, 1211 Geneva 27, Switzerland \\ ${ }^{11}$ Friedrich Loeffler Institute, Seestrasse 55, 16868 Wusterhausen, Germany \\ ${ }^{12}$ Department of Microbiology and Plant Pathology, Faculty of Natural and Agricultural Sciences, University of Pretoria, New \\ Agricultural Building, R9-13, South Africa \\ ${ }^{13}$ Centers for Disease Control and Prevention, 1600 Clifton Road North East, Atlanta, GA, USA \\ ${ }^{14}$ Canadian Food Inspection Agency, 3851 Fallowfield Road, P.O. Box 11300, Station H, Nepean, ON, Canada K2H 8P9
}

Correspondence should be addressed to Tiziana Lembo, tiziana.lembo@glasgow.ac.uk

Received 15 October 2010; Revised 9 February 2011; Accepted 24 February 2011

Academic Editor: Craig Stephen

Copyright ( 2011 Tiziana Lembo et al. This is an open access article distributed under the Creative Commons Attribution License, which permits unrestricted use, distribution, and reproduction in any medium, provided the original work is properly cited.

Canine rabies, responsible for most human rabies deaths, is a serious global public health concern. This zoonosis is entirely preventable, but by focusing solely upon rabies prevention in humans, this "incurable wound" persists at high costs. Although preventing human deaths through canine rabies elimination is feasible, dog rabies control is often neglected, because dogs are not considered typical economic commodities by the animal health sector. Here, we demonstrate that the responsibility of managing rabies falls upon multiple sectors, that a truly integrated approach is the key to rabies elimination, and that considerable progress has been made to this effect. Achievements include the construction of global rabies networks and organizational partnerships; development of road maps, operational toolkits, and a blueprint for rabies prevention and control; and opportunities for scaling up and replication of successful programs. Progress must continue towards overcoming the remaining challenges preventing the ultimate goal of rabies elimination.

\section{Introduction}

Today, RNA viruses play an increasingly important role in emerging human diseases throughout the world [15]. One of the main reasons for this is their ability to evolve rapidly, adapting to new species of hosts and thus to expand their range $[6,7]$, including humans (i.e., new zoonotic diseases). Social and environmental changes also contribute to providing new ecological niches and promoting the rapid selection of novel virus variants [8-10]. This group of viruses includes the causative agents of rabies. As with most generalist agents of a zoonotic nature, rabies is a 
very complex disease, in part because of its wide global distribution, the diverse number of virus variants and host species, its extremely high case fatality rate, and for its existence at the border between animal and human health.

Rabies occurs across Africa, the Americas, Australia, and eastern Europe and Asia, from polar regions to temperate latitudes, and is endemic within tropical areas. Recognized etiological agents consist of an expanding range of species (previously genotypes) of negative-strand RNA viruses of the Lyssavirus genus [11]. Although susceptible natural hosts include all mammals, this zoonosis is perpetuated by domestic and wild carnivores, and by many species of bats, which all act as reservoirs for the eleven proposed Lyssavirus species and for emerging variants within species [12]. Humans are infected after being exposed to virus laden saliva or tissue from a rabid animal, usually through bites into open wounds or contamination of mucous membranes. There is no treatment and rabies almost always leads to death after the onset of clinical symptoms. A plethora of viral species and variants maintained by a diversity of hosts with potential for cross-species and cross-order transfers pose a formidable challenge to a strict concept of true disease eradication [13]. However, in any one geographic area, rabies virus (genotype/serotype/species 1), which is responsible for classical rabies and the most important cause of human cases, is typically maintained within a single terrestrial animal reservoir. Elimination of rabies virus variants is, therefore, a more realistic prospect.

The ultimate objective of controlling rabies is the prevention of human deaths. Human rabies mortality can be reduced through ensuring administration of vaccine and immunoglobulin (postexposure prophylaxis, PEP), following extensive washing of the wound, to all bite victims from infected animals. However, these life-saving biologics are often not accessible or affordable to the populations most at risk [14]. Furthermore, interventions focusing solely on rabies prevention in humans have no impacts on reducing infection in maintenance hosts, hence on achieving rabies elimination from an area-the so-called "incurable wound." For centuries, rabies control in reservoir hosts has constituted an essential measure to decrease the burden of human mortality. Effective vaccines for animals are available, and most developed countries have eliminated canine rabies [1517], responsible for the vast majority (99.9\%) of all human cases [18], by preventing the disease in dogs. However, lack of effective canine rabies prevention and control programs in poorly resourced countries continues to cause tens of thousands of human deaths every year [18]. In canine rabies endemic countries, rabies is a recurrent public health issue and also represents a threat to rabies-free areas through the movement of infected dogs - as was the case in Bali, where rabies was introduced recently $[19,20]$. Despite the availability of modern and efficient prophylaxis tools for humans and animals, on average, rabies still kills one person every 10 minutes, with the highest burden of mortality in Africa and Asia and most of the deaths occurring in children less than 15 years old [18].

Ineffective rabies prevention and control across most of the developing world has been driven by a lack of awareness about disease impacts and institutionalized attitudes about where the responsibility for development and enforcement of rabies control and prevention programs resides. From a public health perspective, rabies remains a "neglected" zoonosis largely because it occurs in communities suffering from poverty and inequitable human and animal health care [21]. Rabies predominantly affects the poorest segments of the population, living in remote, rural areas with insufficient access to life-saving interventions and the services to deliver them. These communities have little political voice and, therefore, limited impact on health policy making. As a consequence, rabies ranks low within national and international agendas, in spite of being present in more than 150 countries/territories and representing a public health threat to more than 3 billion people in Asia and Africa alone [18].

In addition, from an animal health perspective, the species mostly involved in rabies maintenance and transmission to humans in rabies-endemic areas, the domestic dog, is often neglected by veterinary services serving the agricultural sector and/or the public health sector. In Asia, Africa, and Latin America, a substantial proportion of the dog population is unrestricted or semirestricted (stray), neither under direct control of their owners nor confined by a physical barrier [22-28]. This term encompasses both owned and unowned roaming dogs and does not distinguish whether the dog has an "owner" or "guardian". Indeed, in many countries, the majority of dogs defined as roaming are owned, but are allowed to roam on public property for all or part of the day. These dogs fall into an institutional vacuum. They are not included in any conventional health care system as compared to other species recognized as disease vectors (for example, insect vectors of malaria or dengue) that are controlled as part of public health control programs. Dogs are neither a species that the agricultural sector/veterinary services are inclined to take full responsibility for as, unlike livestock species, they are not a recognized economic commodity. Only fully owned and restricted dogs, which are a minority in countries where canine rabies is prevalent, are generally of interest, but mostly to private veterinary practitioners. As a consequence, in many rabies-affected countries, veterinary services and private veterinarians do not have the will nor the desire to prioritize and allocate the resources required to control a disease that is primarily seen as a public health issue with limited impact on production animal health or small animal private practice.

When we consider where the responsibility for rabies control resides, the issue becomes increasingly complex. Given the cosmopolitan nature of rabies, and the widely recognized global burden of mortality among humans, domestic animals, and wildlife $[13,29]$, no one institution, agency, or country can bear the sole responsibility for effective rabies prevention and control. Rather, rabies is a "transboundary" disease, and basic intersectoral cooperation at any level requires a breadth of expertise, not residing in one single health ministry, agricultural department, academic endeavor, or private enterprise. In a true "One Health" context [30], a wide variety of basic disciplines are necessary for a comprehensive rabies elimination program, including animal control and welfare, diagnostic, ecology, economy, 
education, epidemiology, health communication, anthropology, human and animal health services, virology, and wildlife biology. To be truly efficient, such multifunctional programs need to benefit from and begin with the essential support and commitment of country-based human and animal health authorities.

To this effect, significant milestones have been achieved motivated by the compelling need for alleviating the burden of rabies worldwide. Progress has been made towards uniting global expertise to promote a global campaign against rabies, bridging the gap between research-driven knowledge on rabies and its control and practical action towards its elimination, and creating a model that will stimulate further veterinary efforts in global health research and practice towards the control of zoonotic pathogens. Here we illustrate these achievements, including (1) global, "political" initiatives; (2) global, "applied" initiatives; and (3) regional, local, yet scalable and replicable initiatives. We conclude by discussing the challenges and future directions for additional initiatives on a global basis.

\section{Global Partnerships in Rabies Prevention and Control}

2.1. Building Global Partnerships. The building of public private partnerships is proving to be one of the most effective global strategies to address the needs of disadvantaged populations living in the midst of neglected diseases [31] that are no longer considered to be a priority in industrialized nations [32-34]. If established in a balanced manner, public-private partnerships can bring together the power, resources, and experience from both the industrialized and the developing world to create synergies, define priorities, and find solutions. There are many examples of successful public private partnerships that have pooled their resources and have coordinated global strategies to increase the availability of scarce and expensive medical interventions for those that need them most. For example, the Global Alliance to Eliminate Lymphatic Filariasis (GAELF) consists of a partnership between academic and research institutions, advocacy and resource mobilization partners, country representatives, international development agencies and foundations, international organizations, nongovernmental development organizations, and private sector companies [35]. This group retains a "light governance structure" that provides the flexibility to focus on specific needs at regional and national levels [35].

Several attempts at regional or international partnerships in rabies prevention and control have occurred throughout the 20th century. For example, once oral vaccination of wildlife became a reality during the 1980s, the World Health Organization (WHO) held multiple consultations involving its global network of WHO Collaborating Centers for Reference and Research on Rabies for an organized approach towards red fox rabies elimination in Western Europe. The breadth and longevity of several other partnerships, such as the Rabies in the Americas Association, Latin-America National Rabies Directors Network (REDIPRA), Southern and Eastern Africa Rabies Group (SEARG), Rabies in Asia Foundation (RIA), Asia Rabies Expert Bureau (AREB), Africa Rabies Expert Bureau (AFROREB), and the recently created Middle East and Central Eastern Europe Rabies Expert Bureau (MEEREB), all demonstrate the utility of coalitions in successful information flow and regional health promotion in bringing support to local scientific and public health communities worldwide. As consistently observed by all existing rabies networks, basic education of people exposed to the risk of infection, the appropriate training of human and animal health professionals, and necessary advocacy by local health decision makers, are major milestones towards global rabies control. The North American Rabies Management Team is another recent example of professionals in Canada, Mexico, and the United States working together, sharing surveillance data, prioritizing applied research needs, and cooperating across the continent, in training and technology transfer, which allows the implementation of human and animal rabies control programs, achieving a drastic decrease of rabies cases in this region of the world.

The establishment of these partnerships has been of critical importance in raising the profile of rabies at the regional level. However, a much wider (global) approach is needed in strategies aimed at rabies elimination, recognizing that the threat of rabies is evident on every continent throughout the world excluding Antarctica, disregards national borders and that disparity in knowledge and resource capacity should no longer be a reason for inequitable health. In the 21st century, the formation of the Global Alliance for Rabies Control (GARC) and the Partners for Rabies Prevention (PRP) groups perhaps best epitomizes this shift from a regional to a global approach and the modern concept of a global health fellowship [36]. Although these coalitions specifically focus on global rabies control and prevention, they have been established based on a number of broad principles relevant to health issues of zoonotic nature and aimed at addressing the needs of populations suffering from inequitable human and animal health systems. These elements include the following: (1) creating a working group integrating all disciplines/institutions relevant to a comprehensive approach to the control and eventual elimination of a given zoonosis. The inclusion of international organizations is of particular importance, especially as societal and political considerations determine the success of disease elimination [37]; (2) identifying specific gaps concerning biological and technical feasibility, costs and benefits, and societal and political considerations [37], which prevent the control, prevention, and eventual elimination of a given zoonosis; (3) working together to agree upon the most effective strategies to address these gaps; (4) identifying the partners within the partnership that can provide the expertise to deal with these gaps; (5) establishing programs to practically deal with these gaps; and (6) demonstrating success by delivering results with respect to these programs.

GARC is the first global nongovernmental organization focusing specifically on increasing awareness and advocacy for rabies prevention and control by supporting communityled interventions. Using the GAELF as an example, the 
PRP was established in 2008 and is comprised of an informal group of stakeholders working in the field of rabies prevention and control. This is the first time that a working group consisting of all key stakeholders in the field of rabies have joined to find workable solutions to rabies control, including timelines and deliverables. The PRP includes representatives from major international health organizations (WHO/Pan American Health Organization (PAHO), Food and Agriculture Organization (FAO), World Organization for Animal Health (OIE), European Commission (EC)), nongovernmental organizations including GARC and animal welfare organizations, representatives from the human and animal healthcare industry, and global health institutions, such as WHO Collaborating Centers for Rabies Research, and academic institutions working on new rabies prevention and control tools. The individuals in PRP are unpaid volunteers, comprised of the essential disciplines needed towards a common cause of advocacy, action, and research, in an international context. Together, members of the PRP continue to evaluate the global situation of rabies to find feasible, achievable solutions. The PRP thus provides a foundation by which partners can combine their resources, including expertise and experience, communications networks and outreach, and data and educational material to improve access to rabies prevention and control tools (Table 1).

\subsection{Developing and Implementing Programs with Partners.} Public-private partnerships, such as the GAELF and PRP, provide a rich environment for brainstorming and optimizing the skills and knowledge required to generate new ideas, create realistic milestones and deliverables, and build novel systems around which global health strategies can be agreed upon. In the field of rabies, the PRP focuses on increasing global attention on rabies, enhancing educational awareness on all levels of society, and providing new tools for both poorly resourced and industrialized nations to improve access to rabies prevention and control, particularly for those living at greatest risk. Regarding increasing advocacy and awareness on local, national, and international levels, a "road map for rabies prevention and control" was initially developed including short-, medium- and longterm achievable goals. The network of the PRP agreed to utilize their resources to increase educational awareness for rabies prevention and to build a global network of rabies experts by supporting the World Rabies Day initiative, launched in 2007 [38-42]. One example of the successful collaboration provided by the PRP includes the coordinated efforts of the GARC, Centers for Disease Control and Prevention (CDC) in the US, the Universities of Washington and Pretoria, and FAO to distribute over 52,000 rabies awareness posters to 21 countries in Africa. World Rabies Day, observed annually on September 28th, the anniversary of the death of Louis Pasteur, has provided the vehicle by which rabies educational material can be transmitted through the global network set up through the PRP to reach more than 150 countries. Since its inception, lifesaving rabies prevention messages have been sent to more than 150 million people across the globe. The response of communities worldwide since the World Rabies Day inaugural campaign in 2007 is evidence that people living in rabies endemic areas have a desire for relevant educational material and are willing to support community programs aimed at increasing awareness about rabies prevention and control $[40,43]$.

The PRP next combined their knowledge, efforts, and resources to define specific strategies that would enable canine-rabies endemic countries to design their own national programs for preventing human rabies. Despite the availability of several guidelines and publications on various aspects of controlling rabies in dogs and preventing rabies in humans, it was realized that there was no integrated standard operating procedure or "Blueprint" bringing together all necessary aspects for a "One Health" approach to controlling rabies. Rabies is a zoonotic disease and, in order for interventions to be successful, control programs must incorporate an integrated, or One Health, approach including human and animal public health principles, diagnostics and surveillance, education and advocacy, anthropology, social mobilization and community outreach, research and development, and program implementation and evaluation as well as funding options. The need for such comprehensive strategies has been clearly identified following the successful adoption of this approach in Latin America leading to dramatic impacts on human and animal rabies cases [44]. The PRP was able to draw on global expertise to incorporate all of these aspects into a Blueprint for rabies prevention and control which is currently available online. [45]. As a next step, the PRP is focusing on combining their efforts to re-evaluate the global burden of rabies in order to establish the cost effectiveness of intervention strategies.

Operational activities related to public-private partnerships are a challenge because strong partners may be restricted by their own institutions or companies as to their level of involvement and they often do not have the time to dedicate to the administering of required activities to reach agreed upon goals. However, one of the major strengths of a public private partnership is the ability to establish new approaches to overcome administrative barriers. The GARC acts as a secretariat for the PRP and is dedicated to bringing all partners and stakeholders together in a timely manner, without bias, and ensuring that operational activities run smoothly.

Other important considerations involved in setting up public private partnerships include establishing the correct balance between the variety of players that are involved as each may have different and often competing interests. If the correct balance is not initially established or is not continuously maintained, there is a potential to lose focus and the most urgent problems may not be addressed. The PRP has resolved these issues by setting up the organization in a similar manner as the GAELF [35]. The PRP maintains a light governance structure, meeting biannually to discuss global issues regarding rabies prevention and control, identify gaps and how best to address them, and concentrating on finding workable solutions that will benefit all of society (Table 1). 


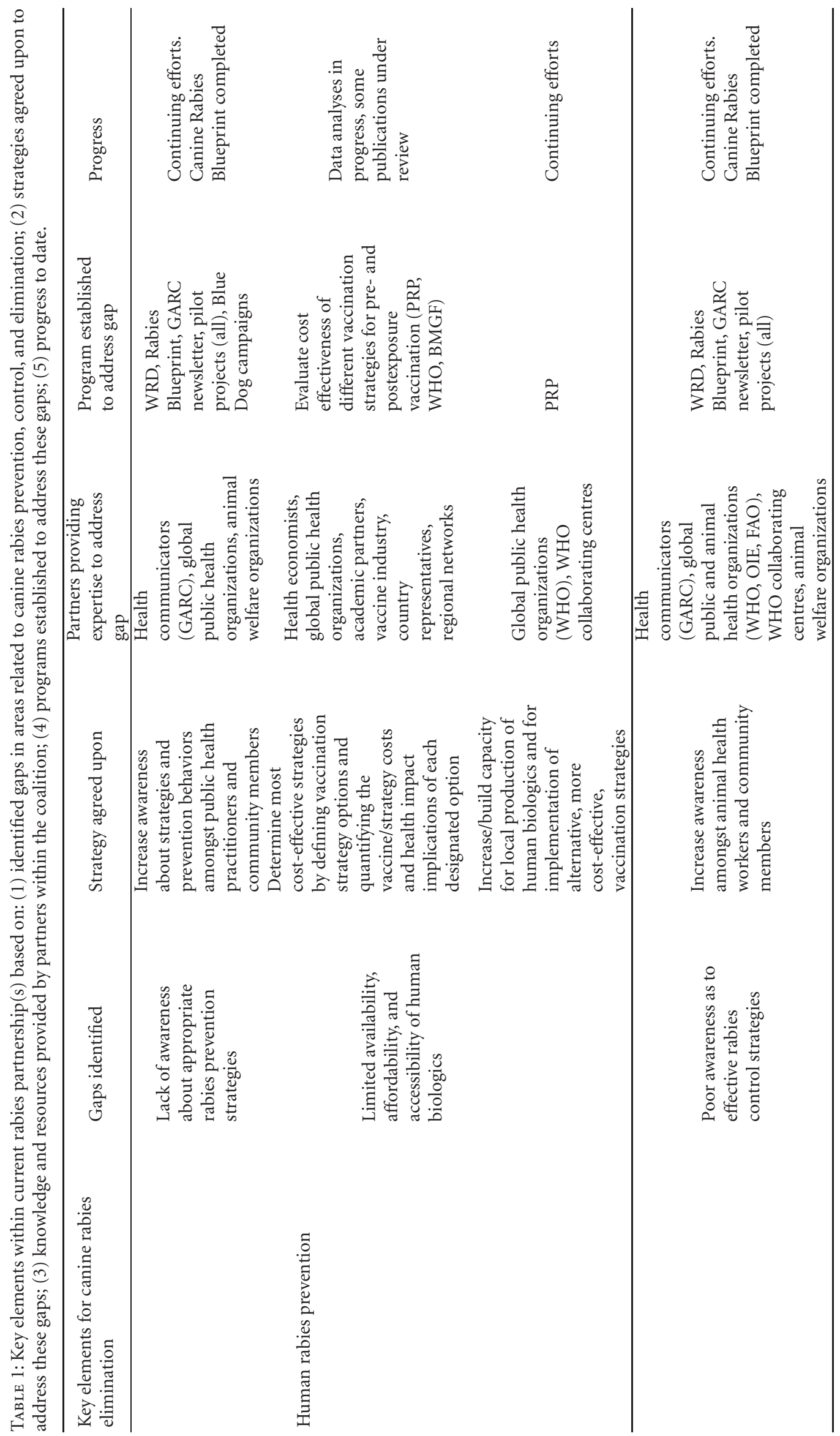




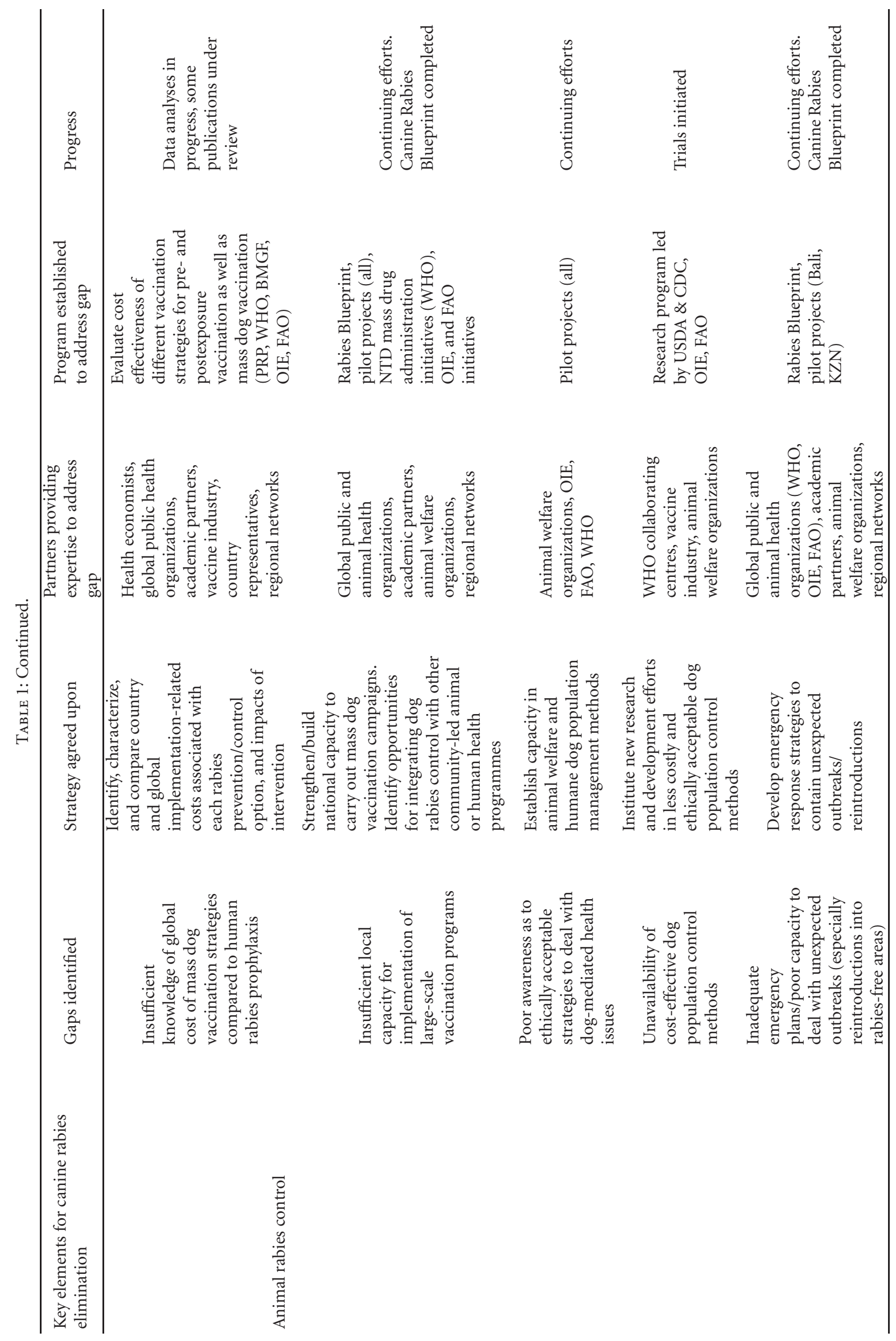




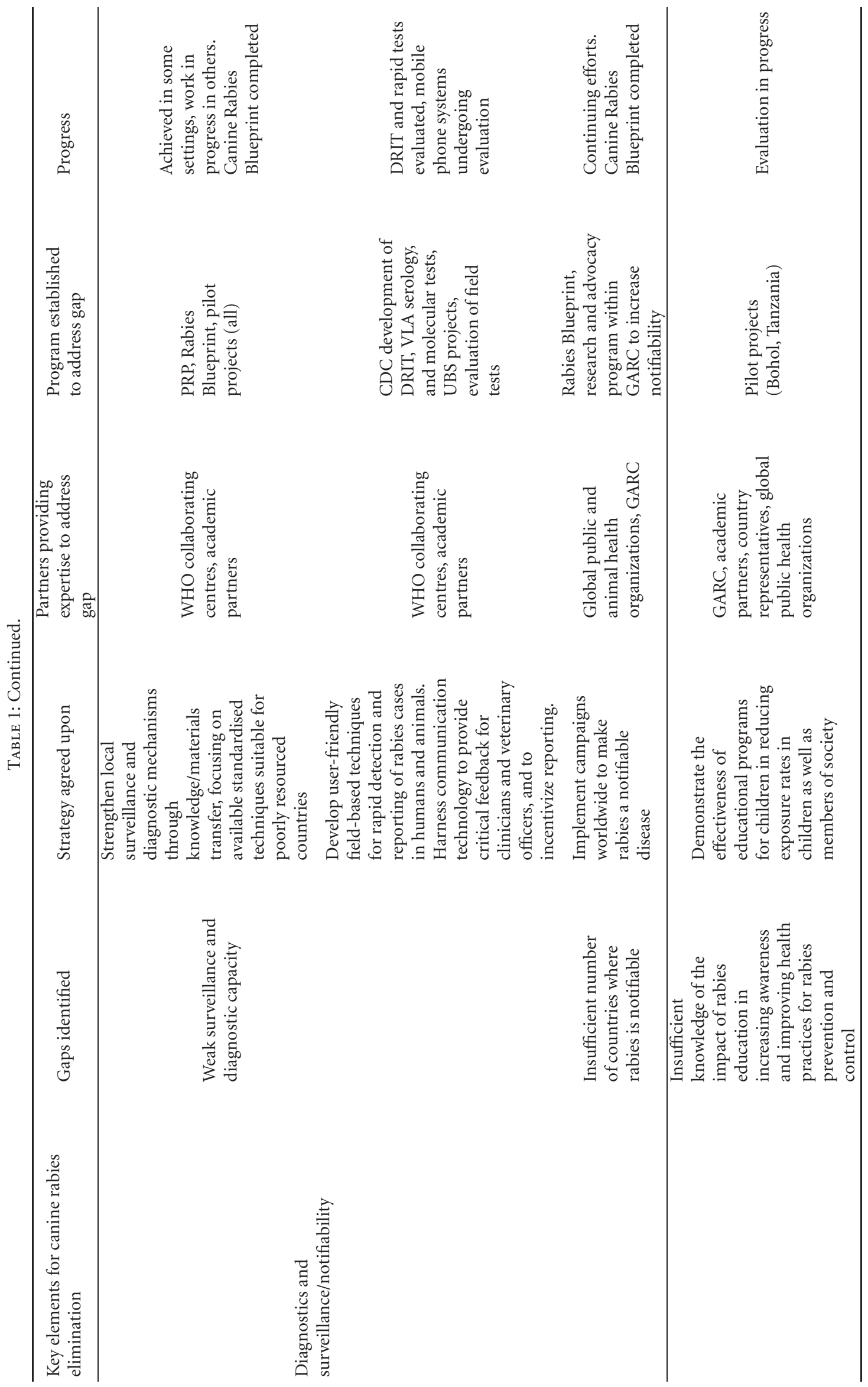




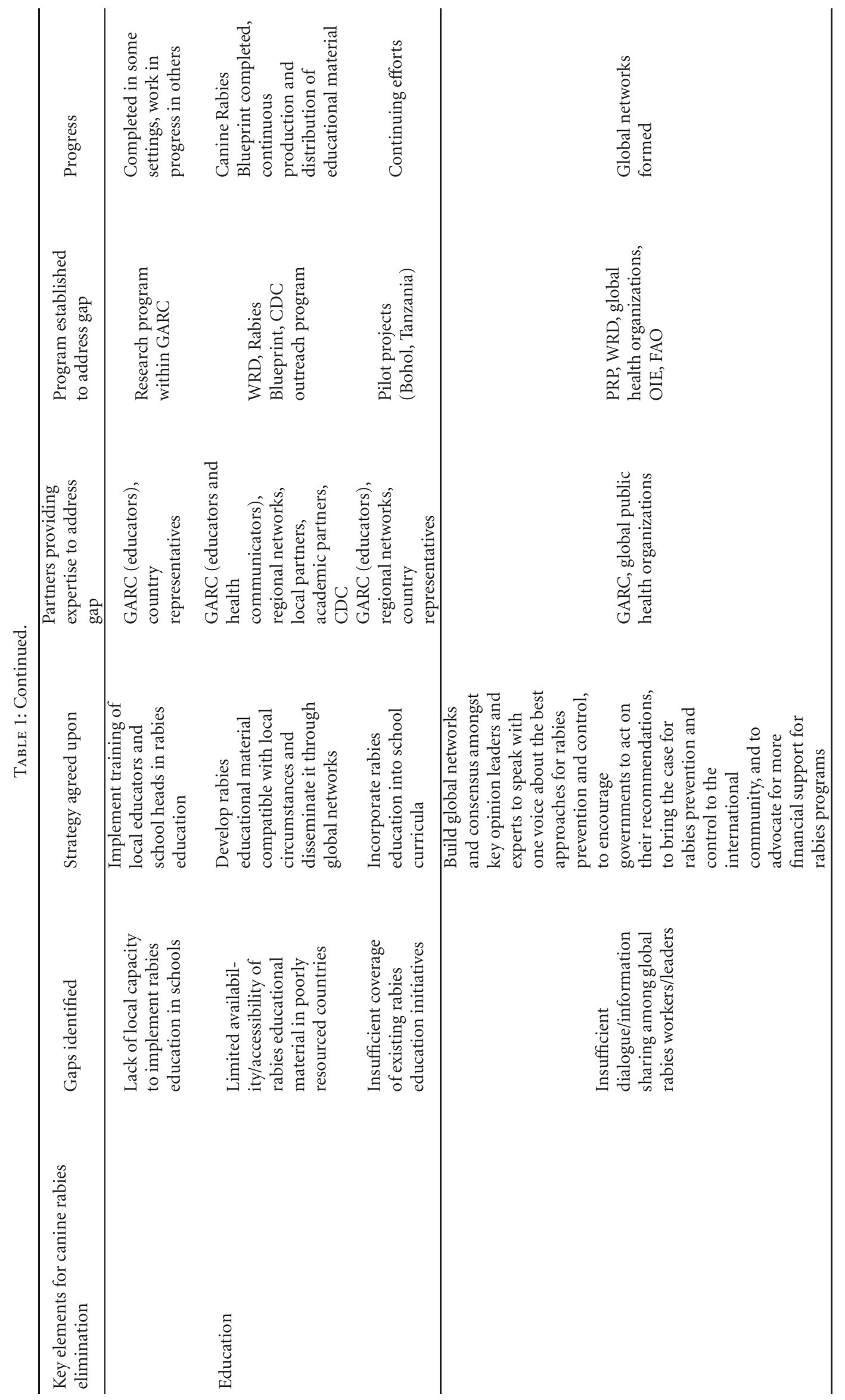




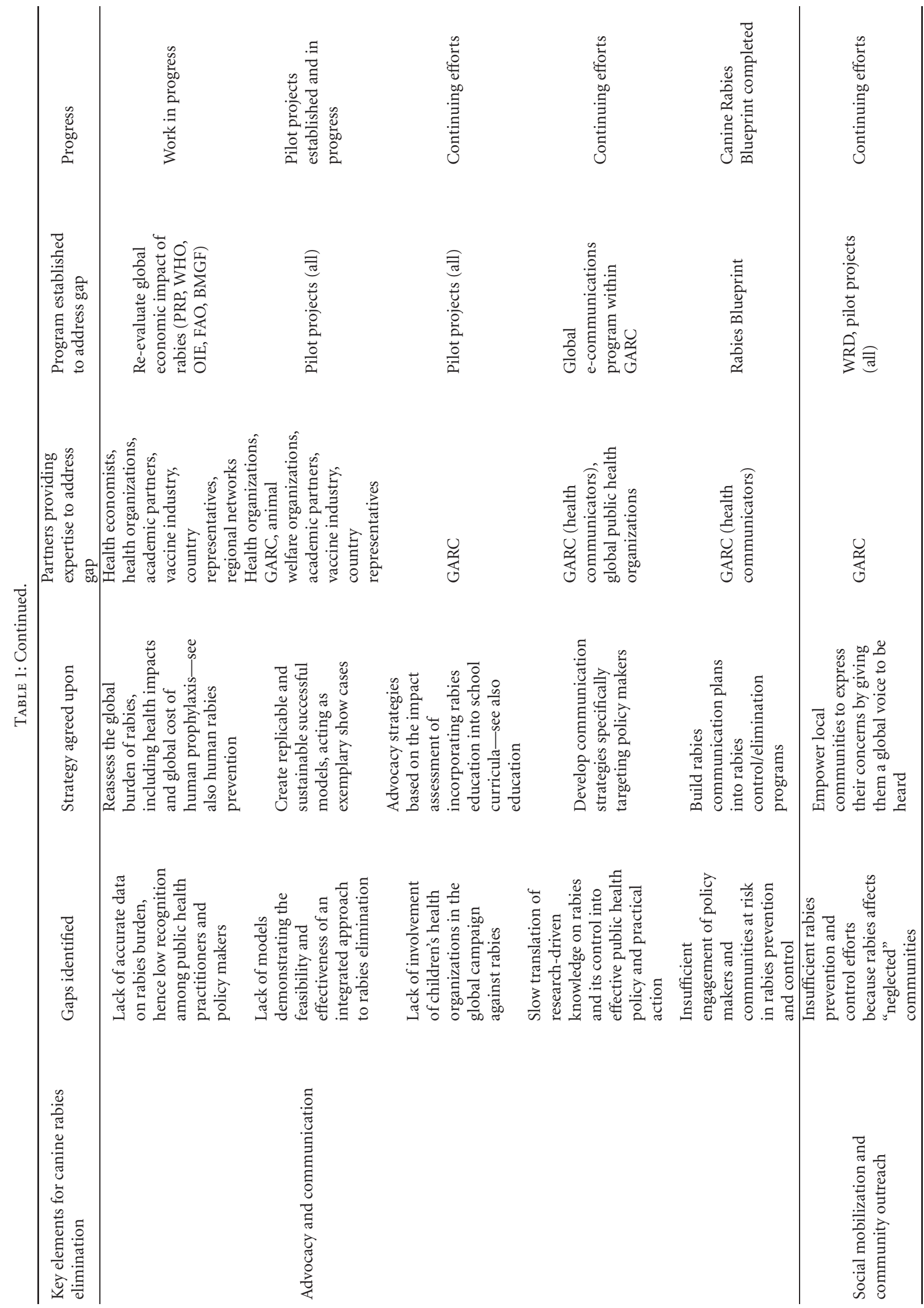




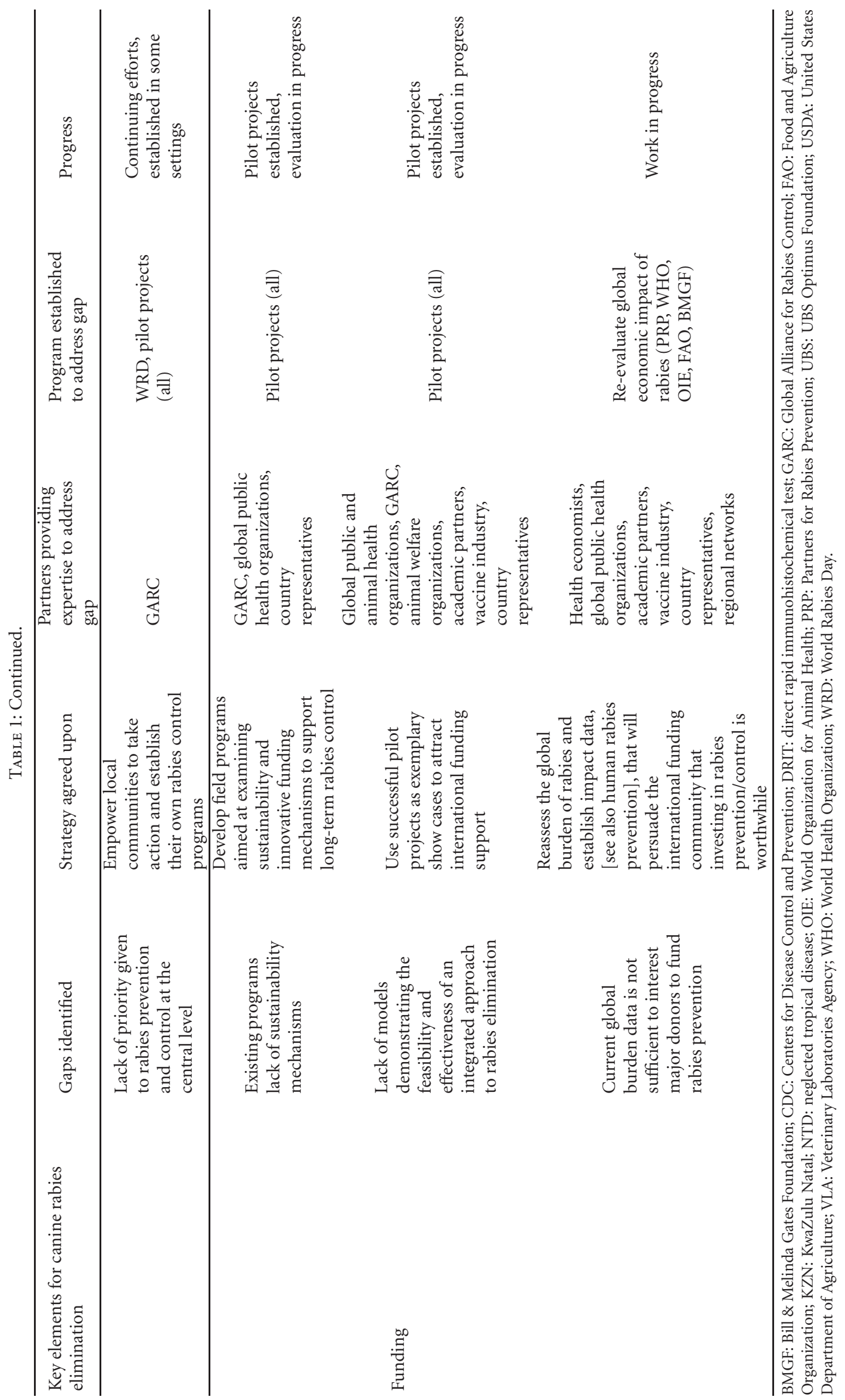




\section{How Can Canine Rabies Be Eliminated?}

3.1. Components of a Canine Rabies Elimination Program. Perhaps the most important insight generated from decades of epidemiological and operational research on rabies is that the elimination of both canine and human rabies is a feasible objective in rabies endemic areas of the less developed world $[12,29,44,46-50]$. We now have a very clear understanding of what constitutes the critical components of a successful rabies elimination program, and we also recognize that a fully integrated approach is critical for achieving this significant goal. One key scientific finding is that where rabies epidemiology is driven by cycles in domestic dogs, as in most of Africa, Asia, and Latin America, well-implemented and sustained rabies vaccination and control programs that target this species will lead to elimination of canine rabies in most areas [12]. Thus, a radical "paradigm shift" in strategic planning and implementation activities is required by the many countries focusing solely on human rabies prophylaxis to prevent human deaths. Dog rabies control should be the focus of efforts and resources also in areas where (occasional) rabies cases are detected in wildlife, since elimination of rabies in dogs should lead to the disappearance of the disease in all other species. Control efforts targeted at wildlife should only be considered where independent transmission cycles are detected in species other than dogs. Once canine rabies has been eliminated, rabies control strategies should aim at maintaining freedom from the disease. A sustainable rabies control and prevention program, therefore, consists of two essential components, an "attack/elimination" component and a "maintenance" component.

Although the implementation of each of these two phases requires specific techniques, a number of "preparatory" steps are essential to both components. These include (1) increasing awareness about the local and global impacts of rabies as well as its burden on public health budgets, and establishing specific roles and responsibilities and cooperation amongst all parties involved in rabies control and prevention activities; (2) building capacity and allocating resources to laboratory-based surveillance, and control and prevention operations, including training of relevant professionals; (3) establishing a legislative framework relevant to rabies control and prevention; (4) ensuring engagement of local communities through raising awareness and education; and (5) obtaining information on the size and accessibility of the dog population to define the best strategy for canine rabies control compatible with local circumstances.

The "attack/elimination" component combines activities aiming at preventing the occurrence of human rabies by reducing virus transmission in the dog population. These include: (1) mass vaccination of the maintenance dog population, (2) management of the dog population, and (3) improved access to wound cleaning necessities, human rabies biologics, and information on available bite centers or emergency rooms. Throughout the elimination component, continuous epidemiological surveillance is essential to monitor the effectiveness of intervention in animal and human populations and to prevent overuse of human biologics.
Mass dog vaccination has long been recognized as the mainstay of successful dog rabies control and eventual elimination $[12,48]$. In the most affected continents, Africa and Asia, generally characterized by very dense dog populations, adequate levels of coverage, of at least $70 \%[12,51]$, as well as sustained and frequent (usually annual) campaigns are essential for effective control aimed at achieving a "rabiesfree" status. Since dogs are often unrestrained and without any apparent evidence of ownership, local authorities often view costly oral vaccination of free-roaming dogs as the only solution to the rabies problem in the reservoir population. However, the vast majority of domestic dog populations have affiliations to households/communities and are, therefore, accessible for central-point parenteral vaccination, considered the most cost-effective strategy [29, 52-55]. Participation in dog vaccination campaigns can also be increased by improved engagement of local communities through education and awareness, as discussed below, and delivery of "primary animal health care" against common infections (mange and internal parasites) to dogs brought to vaccination stations as in KwaZulu-Natal [56]. In some circumstances (e.g., very aggressive or truly unowned dogs, and dispersed communities) more intensive (i.e., house-tohouse) efforts [55] or alternative (oral) delivery strategies $[57,58]$ may, however, be required. In some circumstances, the effectiveness of mass dog vaccination campaigns can be increased through management of the dog population. Needs for this are determined at the start of the program through ecological surveys, generally conducted as part of the preparatory phase. A combination of approaches are available for dog population management programs: promotion of responsible dog ownership through community education and legislative measures (mandatory dog registration and identification, tie-up orders, abandonment legislation, etc.); reproduction control [59]; temporary/permanent removal of dogs (shelters, foster homes, capture and release, euthanasia); and habitat control [60]. Elimination of dogs should be restricted to suspect rabid dogs, unvaccinated contact dogs, and dogs considered unsuitable for rehoming or release at the point of capture, and should always be implemented in conjunction with other approaches [60].

Reducing human rabies incidence through correct utilization of human biologics, including preventive immunization of categories at risk, and wound care (through thorough washing of the wound with water and soap) and prophylaxis (vaccine and rabies immune globulin, RIG, when required) of individuals exposed to suspect rabid animals, is an essential component of the rabies elimination phase. Awareness about prevention behaviors among community members, particularly children, is critical and can be increased through appropriate communication channels, as illustrated below. While the integration of dog rabies control and human rabies prevention approaches is important at the start of the program to reduce animal and human rabies incidence, progressive declines of canine rabies should ultimately result in reduced demand for human biologics [61].

Once freedom from canine rabies [12] has been achieved through the attack/elimination phase, the implementation 
of a "maintenance" phase is required to prevent reintroductions of disease and to ensure a continuous rabiesfree status. This component involves the maintenance of features established in previous phases including continual high political commitment and intersectoral cooperation, surveillance networks to uncover potential new cases or infected areas, regulations for in-country animal movements and animal trade, capacity for dog vaccination (e.g., maintenance/containment/emergency vaccination), policies on the judicious use of human rabies biologics, and educational channels linking government officials and local communities. The maintenance phase also comprises specific activities. An assessment of the rabies situation in neighboring areas is important, ideally followed by the establishment of rabies control and prevention efforts in these jurisdictions through close liaison and effective collaborations involving key stakeholders. Careful consideration also needs to be given to building sustainability in established programs to maintain freedom from rabies in the long term. When national rabies management efforts are developed as multisectoral programs, this could be achieved through integrated financial mechanisms across relevant sectors and parties, with costs and benefits shared across ministries.

Clearly, much progress has been made in the development of tools and strategies required for effective rabies prevention and control. The process of translating researchdriven interventions into better health outcomes for the populations living in rabies-endemic settings is, however, long and difficult. Yet, one recent achievement in this direction is to have gathered and synthesized all current scientific knowledge and available information on rabies prevention and control into the recently launched "Rabies Blueprint" [45], an easy to use operational tool kit, which has the potential to contribute to evidence-based policies and action towards rabies elimination.

3.2. The Role of Global Health Communications. For the successful accomplishment of a canine rabies elimination program, the importance of engaging critical players in rabies prevention and control, that is, national and international policy makers as well as communities at risk, cannot be overstated. A higher level of awareness is a key to effective public and animal health policy and action at all levels, prioritization of rabies in national and international budgets, and increased intersectoral dialogue, hence collaborative rabies prevention and control initiatives. Enhanced awareness on rabies is also essential in preventing and ensuring adequate management of human exposures [12, 62, 63], engaging personnel and communities in rabies prevention and control efforts, and increasing reporting of cases [13].

To this effect, the involvement of global health communications (the study and use of methods to inform and influence individual and community decisions that enhance health) has gained increasing prominence in rabies elimination strategies worldwide. The World Rabies Day initiative is an example of the global impact that health communications can have on rabies prevention and control efforts [38-42]. Utilization of fundamental and innovative communication techniques as part of this initiative has increased awareness globally, enabled life-saving information to be communicated across the world instantaneously, galvanized support, empowered stakeholders at every level, and re-ignited rabies control efforts in countries that had previously abandoned national programs. The establishment of this global rabies network is not the only achievement of including health communications in the global campaign against rabies. Using modern health communications research $[64,65]$, a comprehensive 8-step rabies communications plan, adaptable to the cultural, political, and behavioral needs of any location, has been developed for incorporation into a canine rabies elimination program [45].

Since rabies epidemiology and the behavioral or cultural beliefs of the individuals most at risk will vary across localities, the first step of the communications plan is to identify the important points from an epidemiologic assessment as well as a comprehensive list of potential issues, challenges, and barriers to change that may affect the communication outreach. Challenges may be behavioral (e.g., beliefs on the use of medicine or vaccines), cultural (e.g., perceptions on dogs or other mammalian species), demographic (e.g., economic implications), or physical (e.g., access to healthcare or clean water). Another important aspect is to define the purpose of the communication, including identifying goals and objectives which should be adapted towards national needs. In addition, any outreach should be targeted to specific audiences, who can be segmented according to those most at risk, the primary audience (e.g., children and young boys, who in many areas take care of dogs), and influencers of the primary audience (e.g., healthcare providers, community leaders, and policy makers).

Once draft messages are developed, they should be tested with the target audience. Audience segmentation and message testing will ensure that messages resonate with target populations and can improve uptake of prevention behaviors [66]. In launching a communications campaign, choosing appropriate media channels and determining the best timing for release are also critical. There are numerous ways to disseminate rabies educational messages, and benefits and limitations to each of the channels [67]. After implementing any communications effort, evaluating its impact will help inform and improve future educational outreach, although funding limitations may discourage some localities from undertaking this task. Nonetheless, there are several ways in which rabies programs can be evaluated [68], and options exist for evaluating communication efforts regardless of funding, such as process evaluation and outcome evaluation [45].

\section{Scalable Pilot Projects}

The need for integrated approaches towards human rabies prevention, which incorporate disease control in the canine reservoir, has been widely recognized by major international health organizations. For such strategies to become truly applicable and universally accepted, successful pilot projects are essential to demonstrate the feasibility of canine rabies 
control/elimination and direct benefits for human health. Such projects have the potential to reduce rabies incidence locally, and act as catalysts for encouraging larger-scale or national programs, engaging other areas/countries in similar initiatives, and attracting major international funding support. Building upon the success recognized in Latin America [44], a number of pilot programs have been established recently across Asia and Africa, as described.

4.1. Colombo. A "Humane and Sustainable Dog Population and Management" program has been initiated in Colombo (Sri Lanka) starting in 2007, including the establishment of a formal partnership between three primary partners: Colombo Municipal Council (CMC), Blue Paw Trust (BPT) and the World Society for the Protection of Animals (WSPA). The first phase of the program is planned for 2008-2012. In a second phase (after 2012), CMC will assume primary financial responsibility from WSPA. The current humane program is an evolution of the elimination and pound system of rabies control and dog population management that existed prior to 2007. Key components of the program include mass vaccination and targeted sterilization over the entire spectrum of the dog population; education of children and adults in bite prevention, rabies awareness, and responsible dog ownership; development of "Dog Managed Zones"; and training of CMC staff in relevant skills, including humane dog handling, recognizing dog behavioral signs, delivering responsible ownership messages to communities, and surgical neutering. Increased vaccination and dog management efforts resulting from existing national programs as well as the newly implemented pilot project have led to a considerable reduction in dog rabies cases $[69,70]$. In addition, the combined education program on bite prevention, rabies awareness, and responsible dog ownership has led to greater primary knowledge and its retention. Specifically, $86 \%$ of primary school children and $90 \%$ of secondary school children gained the required knowledge immediately after education sessions, with $85 \%$ of primary and $78 \%$ of secondary school children maintaining this same level of knowledge after 6 months [70].

4.2. Bali. The recent introduction of dog rabies in Bali, Indonesia (with >3.5 million people) during 2008 resulted in a major outbreak, which has killed more than 100 humans with a total of 31,000 dog bite injuries and administration of PEP to 28,000 people since November 2008 [19, 20]. The island had previously been "rabies-free," and the local veterinary authorities had little or no experience in rabies control. First attempts at vaccination and dog movement restrictions failed to stop rabies spread from the initial outbreak on the Bukit peninsular. Following this outbreak, the government began to cull dogs using strychnine, increasing the activity in late 2009. Reasons for this action were that (1) the Bali Animal Husbandry Agency did not believe that reaching sufficient vaccination coverage was possible; (2) vaccination campaigns were inadequate largely because of difficulties in handling many of the dogs, a result in part of the local dog owning culture (hands-off), which is common in most of
Indonesia; and (3) the issue of roaming (stray or outside) dogs caused a distraction, leading to the diversion of limited resources and attention away from effective rabies control via dog vaccination towards attempts to reduce dog populations.

To assist the government in developing a coordinated and effective rabies control program, towards the end of 2009, WSPA started collaborating with local organizations, the Yayasan Bali Animal Welfare Association (BAWA) and the Bali Street Dog Fund Australia, to develop a pilot mass vaccination project in Gianyar, one of Bali's nine regencies. By the end of May 2010, 44,776 dogs had been vaccinated in 537 banjars (banjar = small subvillage of 100-200 dogs approximately), with an estimated $87 \%$ vaccination coverage obtained [69]. Although the average coverage at the end of the first one-day vaccination campaign was $81 \%$, in 81 of the 537 banjars (15\%) the coverage was estimated to be below $70 \%$, and hence a second vaccination campaign was launched. In all cases, second campaigns resulted in over $70 \%$ coverage. While the rate of human rabies deaths has increased recently, with all nine regencies now infected, reflecting the continuation of the epizootic, confirmed human fatalities in Gianyar have been limited to one case since the start of the pilot mass vaccination project [71, 72]. These preliminary results of the pilot program confirm that mass vaccination of the reservoir species is a more effective and humane method of rabies control than culling. In the week before World Rabies Day 2010, the Balinese government signed an agreement with the BAWA to extend the mass vaccination project to Bali's remaining regencies, with cooperation and collaboration of the provincial and regency governments and utilizing a large donation of animal rabies vaccines funded by AusAID and procured by WHO.

4.3. Bohol. Another example of a pilot program for the prevention of human rabies through reservoir control is the Bohol Rabies Program on the provincial island of Bohol with a population of 1.2 million. This program has been anchored on community-based initiatives, focusing on collaboration with the local government, empowerment of local communities to design, implement, and manage their own rabies control programs in accordance with the national rabies program, education of key target audiences (i.e., school children), and elimination of rabies in dogs. The Bohol project has been supported by the GARC and the provincial government, and has also been funded through other cost-sharing activities from the national government, provincial, municipal, and village or barangay local government units, dog owners, and other nongovernmental partners. Since its inception in 2007, the project has mobilized around 15,000 people including local government officers, animal and human health workers, school teachers, village leaders, and volunteers (village-based "rabies watchers"); introduced rabies education into school curricula in all public elementary schools on the island (reaching $>182,000$ children); generated $\$ 105,740$ through community fund schemes to support dog rabies control programs; reduced the dog population by $24 \%$ (including the removal of unregistered, unowned, unmanageable, or unclaimed dogs 
according to the National Animal Welfare Act, and those that had either died of other causes or had been exported from the island); registered 53,692 and vaccinated 53,739 (70\%) dogs; castrated $34.5 \%$ of male dogs; and increased accessibility of PEP (773 in 2005, 1,497 in 2006, 2,906 in 2007, 2,287 in 2008, and 2,276 in 2009) [73]. While the effectiveness of mass dog vaccinations has been enhanced by dog population management in this case, measures are currently being taken to reduce the need to further eliminate dogs and improve animal welfare standards when removal is required. Measurable positive impacts of the program include increased awareness and enhanced animal and human health. Specifically, community awareness surveys revealed that $>94 \%$ of local people heard about rabies, $>61 \%$ had knowledge about rabies transmission, and $>82 \%$ was aware and supportive of the Bohol Rabies Program [73]. Additionally, as of October 2010, Bohol will have had no reported rabies deaths in humans or in animals for two full years.

4.4. Rabies Elimination Demonstration Projects. Rabies projects have also been supported by major international funding agencies to serve as a "hands-on" example to demonstrate the feasibility and effectiveness of an ideal "One Health" approach to human rabies prevention. Through the support of the Bill and Melinda Gates Foundation and coordination by $\mathrm{WHO}$, three large-scale rabies elimination demonstration projects, based on mass vaccination of domestic dogs, have been implemented in Africa and Asia (KwaZulu Natal in South Africa, Tanzania, and Philippines) containing over 33 million people and aiming at vaccinating 3 million dogs [74].

The KwaZulu Natal site (population of 9,500,000 inhabitants) extends from the international borders with Swaziland and Mozambique in the north, to the province of the Eastern Cape in the south, while inland it is bound by the provinces of the Free State and Mpumalanga, and by the Kingdom of Lesotho. The project aims at vaccinating about 700,000 dogs regularly during the project duration. The effective veterinary structure involved in rabies control measures and, more recently, the stronger commitment from provincial authorities alleviated many of the logistical, financial, and managerial difficulties in the implementation and maintenance of successful dog vaccination strategies and have been critical factors in successful implementation to date. Since 2009, the program has already achieved reductions in dog and human rabies prevalence through mass mobile vaccination campaigns, as well as targeted sterilization and dog management programs [56]. The program has also led to notable improvements in the delivery of PEP, with fewer human deaths recorded at present.

In the Philippines, the project includes only the Visayas group of islands covering $25 \%$ of the total number of animal rabies cases, $28 \%$ of the total cases of human rabies, and $27 \%$ of animal bites in the entire country based on the 2006 annual rabies report. Three of the 17 administrative regions of the country, 16 provinces and 31 cities of the country's 82 provinces, and 117 cities are, therefore, included. The project serves almost $19 \%$ of the country's human population (with 17 million inhabitants in the area) and an estimated 2 million dogs.

The project site in south-east Tanzania includes Dar es Salaam, Lindi, Mtwara, Morogoro, and Pwani Regions, comprising 24 districts, 459 wards, with approximately $6,200,000$ people and 400,000 dogs. Infrastructure is being developed, and implementation of prevention and control programs has been initiated in Dar es Salaam and Morogoro regions.

Although the ultimate goal of these programs is to achieve local prevention of human rabies through the elimination of canine rabies from all sites within a 5-year period, the overall project is designed to be extended into neighboring regions and countries in an effort to achieve regional and international success towards broader canine rabies elimination.

\section{Challenges and Future Direction}

There is no doubt that, from a veterinary and medical standpoint, considerable progress has occurred towards rabies prevention and control. Despite this achievement, obstacles remain that have impeded successful control. Here, we discuss these challenges, and suggest future strategies to overcome them.

5.1. Global Burden of Rabies. One of the most important challenges is to reverse the cycle of neglect, which has created a low priority to rabies and its control in many poorly resourced countries. Global advocacy and enhanced awareness of the disease burden among national and international policy makers are essential to improve rabies prevention and control activities. Data on disability-adjusted life year (DALY) scores and economic burden models are required to compare a given condition in relation to other public health issues, although these elements are not the only criteria on which priorities are defined. Evidence-based estimates of the burden of rabies have been developed for Africa and Asia, as well as specific countries, such as Tanzania and Cambodia $[18,75,76]$, which indicate that canine rabies impacts human and animal health substantially, as well as local and national economies and wildlife conservation [29]. The global burden of rabies, including a more accurate evaluation of the global cost of postexposure prophylaxis as well as mass dog vaccination strategies, needs to be further documented and brought into the spotlight, particularly to the attention of national policy makers in rabies-affected countries and to the international health community. Therefore, there is an urgent need to gain a better sense of the burden of animal and human rabies in any given country. Given that poor surveillance and underreporting have dramatically contributed to a lack of data on disease impacts [77], a focus on strengthening national capacity to better diagnose rabies and investigate human cases and animal outbreaks is essential for effective disease surveillance and a more accurate burden assessment. 
5.2. Veterinary Services. Clearly, rabies is a primary public health issue, but is not without agricultural impacts. Major obstacles are the lack of priority given by the agricultural sector to the control of animal health problems unrelated to livestock species and poor awareness as to effective and ethically acceptable strategies to deal with dog-mediated health issues. This lack of priority is partially due to misconceptions about whose responsibility rabies control is. Veterinary services may usually handle dog-related problems in extreme situations (e.g., to "clean up" areas ahead of specific events, respond to complaints by the public about nuisance caused by "stray" dogs, including bites, or respond to major rabies outbreaks), usually through indiscriminate catch-and-kill operations. These operations are unpopular at the local and international level and counterproductive in the short and long terms. In addition, national and/or local organizations promoting animal welfare and responsible pet ownership, often supported by international animal welfare organizations (e.g., WSPA, Humane Society International, International Fund for Animal Welfare, etc.), are becoming more influential in an increasing number of poorly resourced countries. In many instances, these nongovernmental organizations will oppose catch- and-kill municipality actions, on ethical grounds and also on the basis of lack of effectiveness of these approaches, using evidence-based recommendations of humane alternatives $[12,60]$.

In any plan for rabies prevention and control, there is a need to bring all disparate partners (national human health and animal health services, municipality services, nongovernmental organizations, etc.) together in a collegial, rather than adversarial fashion. In addition, there is a need to provide support for veterinary services to carry out annual or biannual mass dog vaccination campaigns for the multiple years required to eliminate dog rabies, particularly given other priorities relating to livestock production. As demonstrated in many Latin American countries, and in Bali and some Indian states, where the leading role in rabies control is assumed by the public health sector and local, national, and international animal welfare organizations, respectively, the additional intersectoral support provided to veterinary services can greatly improve dog rabies control program design, implementation, and sustainability [59].

5.3. Human Prophylaxis. Rabies impact is mostly borne by bite victims and the health care system. In many countries the public health sector is often the first to advocate for dog rabies control, as it is usually responsible for the financial burden, in terms of health care and biologics costs. This sector has to assume responsibility for human rabies deaths following exposures to rabid animals when control strategies in the animal reservoir are not effectively implemented and when PEP is not administered correctly. In addition, the government and the Ministry of Health should supply necessary care and biologics to prevent the occurrence of rabies. Provision of vaccines and RIG needed to prevent rabies deaths is estimated theoretically to be around 200 $\mathrm{PEP} / 100,000$ inhabitants (based on figures from a range of poorly resourced countries) [78], although this is likely to vary depending on the setting. While rabies vaccine costs remain in the range of other public health program expenses supported by national health services in several countries, in the absence of international funding these resources are usually beyond the capacity of most impoverished countries. These life-saving products are absolutely necessary and for obvious ethical reasons the considerable sums required to acquire them cannot be diverted from their emergency use. However, improvements in the long-term cost effectiveness of human rabies prevention will only be achieved by focusing on control of rabies in its animal reservoir. Yet, funds initially required to acquire human biologics cannot be diverted to another sector to help increase its capacity to establish effective animal rabies control programs [52, 54, $79,80]$. Any country willing to embark in rabies control initiatives must realize that additional investment will be required in all sectors, and that savings, particularly through reduced demand for PEP, will only occur after several years of implementation. Therefore, it is essential in this initial investment phase that resources can be mobilized among all sectors for human rabies prevention and canine rabies elimination.

Concerning human prophylaxis, an additional challenge is the lack of or limited availability and accessibility of human biologics for PEP, a reoccurring problem in many poorly resourced countries. These products should be provided free or at a subsidized price by the health care system, but in many places they are sold by government-owned and private clinics at a cost, which is often beyond the financial means of animal bite victims. The full cost of PEP alone (direct costs of vaccine and RIG range between $\$ 40$ to $\$ 75$, depending on country and product used [18]) as well as additional costs (medical fees, transportation to hospital, accommodation, and income loss) can easily represent several months of a rural family revenue $[18,29]$. Rabies biologics costs have been decreasing gradually mostly due to a more competitive market, with new vaccines and RIG being produced by manufacturers based in emerging economies (particularly in India and China). Although only a small number of vaccines are currently prequalified by $\mathrm{WHO}$, more emerging products should fulfill WHO requirements and obtain this recognition, increasing market competition and further reducing costs. However, any increase in the availability of additional products must be balanced against the risk of biologics having poor quality [81] or counterfeit origins by greater regulatory scrutiny. To assist dog-bite victims and governments struggling to provide adequate quantities of rabies biologics locally, WHO has been promoting the use of the intradermal route for PEP, which provides a reduction by 60 to $80 \%$ of the vaccine needed for one PEP regimen [82]. Adoption of the intradermal route should be further encouraged [83]. Furthermore, research on new products, especially cocktails of monoclonal antibodies [84] to replace human and equine RIG, improved vaccines, reduced PEP regimens, and intradermal delivery devices should be a priority [59]. 


\section{Conclusions}

Eradication of a disease (e.g., rabies) brings the greatest health benefit, which is the absence of the health threat. It is also the quintessential example of health equity, as all mankind reaps the benefits, leading to eternal cost savings. Rabies is undoubtedly the most feared zoonosis in the world, and takes a heavy toll on underprivileged communities living in the poorest countries. The expertise to reduce the global burden of rabies has been built, and significant strides have been made scientifically and practically towards eliminating canine rabies and reducing human rabies deaths in the industrialized world. Moreover, considerable milestones have been achieved towards taking this progress to the next level, by reducing the dramatic impact of rabies on human and animal communities in endemic areas of the developing world. In an effort to enact a "One Health" approach towards global rabies elimination, the creation of effective partnerships has focused on coordinating research and operational activities related to canine rabies elimination. A global rabies network, covering over 150 countries, and uniting stakeholders from the spectrum of veterinary, public health, pharmaceutical, animal welfare, and wildlife conservation agencies, as well as local communities, has been established. Strategies and tools to successfully prevent and control rabies have been formulated to assist rabies-affected areas in management efforts. Operational activities have been implemented in a diversity of Asian, African, and Latin American settings to create replicable and sustainable models, acting as exemplary showcases for others. More broadly, current strategies towards rabies elimination have the potential to be used as a modern example of integrated, evidence-driven, research-based approaches applicable to other major zoonotic pathogens affecting global health. While access to tools and knowledge has been increased, providing all countries with the potential to eliminate rabies from their territories, the ultimate achievement of this goal, which would lead to health equity, continues to face many challenges. Political and financial constraints remain, highlighting the need for further action towards building the political will to eliminate rabies and to create sustainable and economic solutions, to develop additional practical research tools, and to attract long-term national and international funding support.

\section{Acknowledgments}

The authors would like to thank Federico Spinola for rendering productive and enjoyable reunions possible, and Kim Doyle for stimulating discussions and continuous support. The findings and conclusions in this report are those of the authors and do not necessarily represent the views of the Centers for Disease Control and Prevention.

\section{References}

[1] R. G. Bengis, F. A. Leighton, J. R. Fischer, M. Artois, T. Mörner, and C. M. Tate, "The role of wildlife in emerging and reemerging zoonoses," Revue Scientifique et Technique de l'OIE, vol. 23, no. 2, pp. 497-511, 2004.
[2] S. Cleaveland, M. K. Laurenson, and L. H. Taylor, "Diseases of humans and their domestic mammals: pathogen characteristics, host range and the risk of emergence," Philosophical Transactions of the Royal Society of London Series B, vol. 356, no. 1411, pp. 991-999, 2001.

[3] S. J. Cutler, A. R. Fooks, and W. H. M. van der Poel, "Public health threat of new, reemerging, and neglected zoonoses in the industrialized world," Emerging Infectious Diseases, vol. 16, no. 1, pp. 1-7, 2010.

[4] F. X. Meslin, K. Stöhr, and D. Heymann, "Public health implications of emerging zoonoses," Revue Scientifique et Technique de l'OIE, vol. 19, no. 1, pp. 310-317, 2000.

[5] L. H. Taylor, S. M. Latham, and M. E. J. Woolhouse, "Risk factors for human disease emergence," Philosophical Transactions of the Royal Society B, vol. 356, no. 1411, pp. 983989, 2001.

[6] D. S. Burke, "Evolvability of emerging viruses," in Pathology of Emerging Infections 2, A. M. Nelson and C. R. Horsburgh, Eds., pp. 1-12, American Society of Microbiology, Washington, DC, USA, 1998.

[7] M. E. J. Woolhouse, D. T. Haydon, and R. Antia, "Emerging pathogens: the epidemiology and evolution of species jumps," Trends in Ecology \& Evolution, vol. 20, no. 5, pp. 238-244, 2005.

[8] E. C. Holmes, "The phylogeography of human viruses," Molecular Ecology, vol. 13, no. 4, pp. 745-756, 2004.

[9] E. G. Holmes and A. Rambaut, "Viral evolution and the emergence of SARS coronavirus," Philosophical Transactions of the Royal Society B, vol. 359, no. 1447, pp. 1059-1065, 2004.

[10] T. Kuiken, R. Fouchier, G. Rimmelzwaan, and A. Osterhaus, "Emerging viral infections in a rapidly changing world," Current Opinion in Biotechnology, vol. 14, no. 6, pp. 641-646, 2003.

[11] ICTV, "International Committee on Taxonomy of VirusesIndex of viruses," August 2010, http://www.ictvonline.org/ index.asp? bhcp $=1$.

[12] WHO, "WHO expert consultation on rabies," WHO Technical Report Series, vol. 931, pp. 1-88, 2005.

[13] C. E. Rupprecht, J. Barrett, D. Briggs et al., "Can rabies be eradicated?" in Towards the Elimination of Rabies in Eurasia, B. Dodet, A. R. Fooks, T. Muller, and N. Tordo, Eds., vol. 131, pp. 95-121, 2008.

[14] K. Hampson, A. Dobson, M. Kaare et al., "Rabies exposures, post-exposure prophylaxis and deaths in a region of endemic canine rabies," PLoS Neglected Tropical Diseases, vol. 2, no. 11, e339, 2008.

[15] CDC, "US declared canine-rabies free," August 2010, http:// www.cdc.gov/news/2007/09/canine_rabies.html.

[16] OIE, Historical Perspective of Rabies in Europe and the Mediterranean Basin, World Organization for Animal Health (OIE), Paris, France, 2004.

[17] A. Velasco-Villa, S. A. Reeder, L. A. Orciari et al., "Enzootic rabies elimination from dogs and reemergence in wild terrestrial carnivores, United States," Emerging Infectious Diseases, vol. 14, no. 12, pp. 1849-1854, 2008.

[18] D. L. Knobel, S. Cleaveland, P. G. Coleman et al., "Reevaluating the burden of rabies in Africa and Asia," Bulletin of the World Health Organization, vol. 83, no. 5, pp. 360-368, 2005.

[19] BaliDiscoveryTours, October 2010, http://www.balidiscovery .com/. 
[20] CDC, "Rabies in Bali, Indonesia," October 2010, http:// wwwnc.cdc.gov/travel/content/outbreak/rabies-bali-indonesia2008.aspx.

[21] A. R. Fooks, "Rabies remains a 'neglected disease," Euro Surveillance, vol. 10, no. 11, pp. 211-212, 2005.

[22] U. Kayali, R. Mindekem, N. Yémadji et al., "Coverage of pilot parenteral vaccination campaign against canine rabies in N'Djaména, Chad," Bulletin of the World Health Organization, vol. 81, no. 10, pp. 739-744, 2003.

[23] W. Kongkaew, P. Coleman, D. U. Pfeiffer, C. Antarasena, and A. Thiptara, "Vaccination coverage and epidemiological parameters of the owned-dog population in Thungsong District, Thailand," Preventive Veterinary Medicine, vol. 65, no. 1-2, pp. 105-115, 2004.

[24] B. D. Perry, "Dog ecology in eastern and southern Africa: implications for rabies control," Onderstepoort Journal of Veterinary Research, vol. 60, no. 4, pp. 429-436, 1993.

[25] M. Ratsitorahina, J. H. Rasambainarivo, S. Raharimanana et al., "Dog ecology and demography in Antananarivo, 2007," BMC Veterinary Research, vol. 5, p. 21, 2009.

[26] L. E. Robinson, M. E. Miranda, N. L. Miranda, and J. E. Childs, "Evaluation of a canine rabies vaccination campaign and characterization of owned-dog populations in the Philippines," Southeast Asian Journal of Tropical Medicine and Public Health, vol. 27, no. 2, pp. 250-256, 1996.

[27] K. Suzuki, J. A. C. Pereira, L. A. Frías, R. López, L. E. Mutinelli, and E. R. Pons, "Rabies-vaccination coverage and profiles of the owned-dog population in Santa Cruz de la Sierra, Bolivia," Zoonoses and Public Health, vol. 55, no. 4, pp. 177-183, 2008.

[28] A. I. Wandeler, H. C. Matter, A. Kappeler, and A. Budde, "The ecology of dogs and canine rabies: a selective review," Revue Scientifique et Technique de l'OIE, vol. 12, no. 1, pp. 51-71, 1993.

[29] T. Lembo, K. Hampson, M. T. Kaare et al., "The feasibility of canine rabies elimination in Africa: dispelling doubts with data," PLoS Neglected Tropical Diseases, vol. 4, no. 2, e626, 2010.

[30] A. R. Fooks, "Rabies-the need for a 'one medicine' approach,” Veterinary Record, vol. 161, no. 9, pp. 289-290, 2007.

[31] WHO, Working to overcome the global impact of neglected tropical diseases. First WHO report on neglected tropical diseases, World Health Organization, Geneva, Switzerland, 2010.

[32] USAID, “Global partnerships," August 2010, http://www.usaid .gov/our_work/global_partnerships/.

[33] WHO, "The control of neglected zoonotic diseases. A route to poverty alleviation. Report of a joint WHO/DFID-AHP meeting with the participation of FAO and OIE, Geneva September 2005," 2006.

[34] WHO, "Integrated control of neglected zoonotic diseases in Africa. Applying the 'One Health' concept. Report of a joint WHO/EU/ILRI/DBL/FAO/OIE/AU meeting, ILRI Headquarters, Nairobi November 2007," 2008.

[35] GAELF, "Global Alliance to Eliminate Lymphatic Filariasis. Partners," October 2010, http://www.filariasis.org/who_we_are/partners.html.

[36] ARC, "Partners for Rabies Prevention," August 2010, http:// www.rabiescontrol.net/EN/prp.html.

[37] B. Aylward, K. A. Hennessey, N. Zagaria, J. M. Olivé, and S. Cochi, "When is a disease eradicable? 100 years of lessons learned," American Journal of Public Health, vol. 90, no. 10, pp. 1515-1520, 2000.

[38] D. Briggs and C. A. Hanlon, "World Rabies Day: focusing attention on a neglected disease," Veterinary Record, vol. 161, no. 9, pp. 288-289, 2007.
[39] S. Cleaveland, P. Costa, T. Lembo, and D. Briggs, "Catalysing action against rabies," Veterinary Record, vol. 167, no. 11, pp. 422-423, 2010.

[40] P. Costa, D. J. Briggs, A. Tumpey, R. Dedmon, and J. Coutts, "World Rabies Day outreach to Asia: empowering people through education," Asian Biomedicine, vol. 3, no. 4, pp. 451457, 2009.

[41] B. Dodet and AREB, "An important date in rabies history," Vaccine, vol. 25, no. 52, pp. 8647-8650, 2007.

[42] None, "Rabies has its day," Lancet Infectious Diseases, vol. 7, no. 10, p. 631, 2007.

[43] P. Costa, D. Briggs, and R. Dedmon, "World Rabies Day (September 28, 2010): the continuing effort to 'make rabies history," Asian Biomedicine, vol. 4, p. 671, 2010.

[44] M. C. Schneider, A. Belotto, M. P. Adé et al., "Current status of human rabies transmitted by dogs in Latin America," Cadernos de Saúde Pública, vol. 23, no. 9, pp. 2049-2063, 2007.

[45] PRP, "Blueprint for rabies prevention and control," August 2010, http://www.rabiesblueprint.com/.

[46] K. Hampson, J. Dushoff, S. Cleaveland et al., "Transmission dynamics and prospects for the elimination of canine rabies," PLoS Biology, vol. 7, no. 3, e53, 2009.

[47] WHO, Guidelines for Dog Rabies Control, World Health Organization, Geneva, Switzerland, 1987.

[48] WHO, "WHO Expert Committee on rabies, Geneva September 1991: eight report," WHO Technical Report Series, vol. 824, pp. 1-84, 1992.

[49] WHO, "Strategies for the control and elimination of rabies in Asia. Report of a WHO interregional consultation, July 2001," WHO/CDS/CSR/EPH/2002.8, World Health Organization, Geneva, Switzerland, 2001.

[50] WHO and F. Merieux, "Rabies control in Asia," in Proceedings of the fourth international symposium on rabies control in Asia, John Libbey Eurotext, Hanoi, Viet Nam, March 2001.

[51] P. G. Coleman and C. Dye, "Immunization coverage required to prevent outbreaks of dog rabies," Vaccine, vol. 14, no. 3, pp. 185-186, 1996.

[52] J. Zinsstag, S. Dürr, M. A. Penny et al., "Transmission dynamics and economics of rabies control in dogs and humans in an African city," Proceedings of the National Academy of Sciences of the United States of America, vol. 106, no. 35, pp. 14996-15001, 2009.

[53] J. Zinsstag, E. Schelling, F. Roth, B. Bonfoh, D. de Savigny, and M. Tanner, "Human benefits of animal interventions for zoonosis control," Emerging Infectious Diseases, vol. 13, no. 4, pp. 527-531, 2007.

[54] K. Bogel and F. X. Meslin, "Economics of human and canine rabies elimination: guidelines for programme orientation," Bulletin of the World Health Organization, vol. 68, no. 3, pp. 281-291, 1990.

[55] M. Kaare, T. Lembo, K. Hampson et al., "Rabies control in rural Africa: evaluating strategies for effective domestic dog vaccination," Vaccine, vol. 27, no. 1, pp. 152-160, 2009.

[56] K. Le Roux, "Report to the international coordinating group of the WHO/Gates Foundation funded project on human rabies prevention through dog rabies control and eventual elimination. Dar es Salaam, Tanzania October 2010," 2010.

[57] R. Estrada, A. Vos, R. De Leon, and T. Mueller, "Field trial with oral vaccination of dogs against rabies in the Philippines," BMC Infectious Diseases, vol. 1, 23, 2001.

[58] WHO, Oral vaccination of dogs against rabies. Guidance for research on oral rabies vaccines and field application of oral vaccination of dogs against rabies, World Health Organization, Geneva, Switzerland, 2007. 
[59] WHO, "Human and dog rabies prevention and control. Report of the WHO/Bill \& Melinda Gates Foundation Consultation. Annecy, France October 2009," 2010.

[60] ICAM, "Humane dog population management guidance," International Companion Animal Management Coalition, 2007.

[61] S. Cleaveland, M. Kaare, P. Tiringa, T. Mlengeya, and J. Barrat, "A dog rabies vaccination campaign in rural Africa: impact on the incidence of dog rabies and human dog-bite injuries," Vaccine, vol. 21, no. 17-18, pp. 1965-1973, 2003.

[62] B. Dodet, A. Goswami, A. Gunasekera et al., "Rabies awareness in eight Asian countries," Vaccine, vol. 26, no. 50, pp. 63446348, 2008.

[63] G. C. Matibag, Y. Ohbayashi, K. Kanda et al., "A pilot study on the usefulness of information and education campaign materials in enhancing the knowledge, attitude and practice on rabies in rural Sri Lanka," Journal of Infection in Developing Countries, vol. 3, no. 1, pp. 55-64, 2009.

[64] R. C. Brownson and D. E. Nelson, Communicating Public Health Information Effectively: A Guide for Practitioners, American Public Health Association, Washington, DC, USA, 2002.

[65] NCI, Managing Health Communication Programs Work, Department of Health and Human Services, Washington, DC, USA, 2008.

[66] M. D. Slater, "Theory and method in health audience segmentation," Journal of Health Communication, vol. 1, no. 3, pp. 267-283, 1996.

[67] PRP, "Communication channels," August 2010, http://www .rabiesblueprint.com/spip.php?article143.

[68] CDC, "Framework for program evaluation in public health," Morbidity and Mortality Weekly Report, vol. 48, pp. 1-40, 1999.

[69] WHO, "Rabies elimination in South-East Asia. Report of a workshop. Colombo, Sri Lanka, November 2005,” World Health Organization. Regional Office for South-East Asia, New Delhi SEA-HLM-389, 2005.

[70] WSPA, "Humane and sustainable dog population management in Colombo; mid-project review June 2007-June 2010," London, UK, 2010.

[71] ProMed-Mail, "Gianyar man dies of rabies," ProMed, October 2010, http://www.promedmail.org/.

[72] WSPA, "Case study; Gianyar's mass vaccination project," London, UK, 2010.

[73] ARC, "Alliance for Rabies Control—Projects overviewPhilippines," August 2010, http://www.rabiescontrol.net/EN/ Programs/Projects-Overview/philippines.html.

[74] WHO, "Bill \& Melinda Gates Foundation fund WHOcoordinated project to control and eventually eliminate rabies in low-income countries," October 2010, http://www.who .int/rabies/bmgf_who_project/en/index.html.

[75] S. Cleaveland, E. M. Fèvre, M. Kaare, and P. G. Coleman, "Estimating human rabies mortality in the United Republic of Tanzania from dog bite injuries," Bulletin of the World Health Organization, vol. 80, no. 4, pp. 304-310, 2002.

[76] S. Ly, P. Buchy, N. Y. Heng et al., "Rabies situation in Cambodia," PLoS Neglected Tropical Diseases, vol. 3, no. 9, e511, 2009.

[77] M. Mallewa, A. R. Fooks, D. Banda et al., "Rabies encephalitis in malaria-endemic area, Malawi, Africa," Emerging Infectious Diseases, vol. 13, no. 1, pp. 136-139, 2007.

[78] K. Bogel and E. Motschwiller, "Incidence of rabies and postexposure treatment in developing countries," Bulletin of the World Health Organization, vol. 64, no. 6, pp. 883-887, 1986.
[79] S. Durr, R. Mindekem, Y. Kaninga et al., "Effectiveness of dog rabies vaccination programmes: comparison of ownercharged and free vaccination campaigns," Epidemiology and Infection, vol. 137, no. 11, pp. 1558-1567, 2009.

[80] U. Kayali, R. Mindekem, G. Hutton, A. G. Ndoutamia, and J. Zinsstag, "Cost-description of a pilot parenteral vaccination campaign against rabies in dogs in N’Djaména, Chad," Tropical Medicine and International Health, vol. 11, no. 7, pp. 1058-1065, 2006.

[81] R. L. Hu, A. R. Fooks, S. F. Zhang, Y. Liu, and F. Zhang, "Inferior rabies vaccine quality and low immunization coverage in dogs (Canis familiaris) in China," Epidemiology and Infection, vol. 136, no. 11, pp. 1556-1563, 2008.

[82] M. J. Warrell, A. Riddell, L. M. Yu et al., "A simplified 4-site economical intradermal post-exposure rabies vaccine regimen: a randomised controlled comparison with standard methods," PLoS Neglected Tropical Diseases, vol. 2, no. 4, e224, 2008.

[83] WHO, "Rabies vaccines: WHO position paper," Weekly Epidemiological Record, vol. 85, pp. 309-320, 2010.

[84] T. Müller, B. Dietzschold, H. Ertl et al., "Development of a mouse monoclonal antibody cocktail for post-exposure rabies prophylaxis in humans," PLoS Neglected Tropical Diseases, vol. 3, no. 11, e542, 2009. 

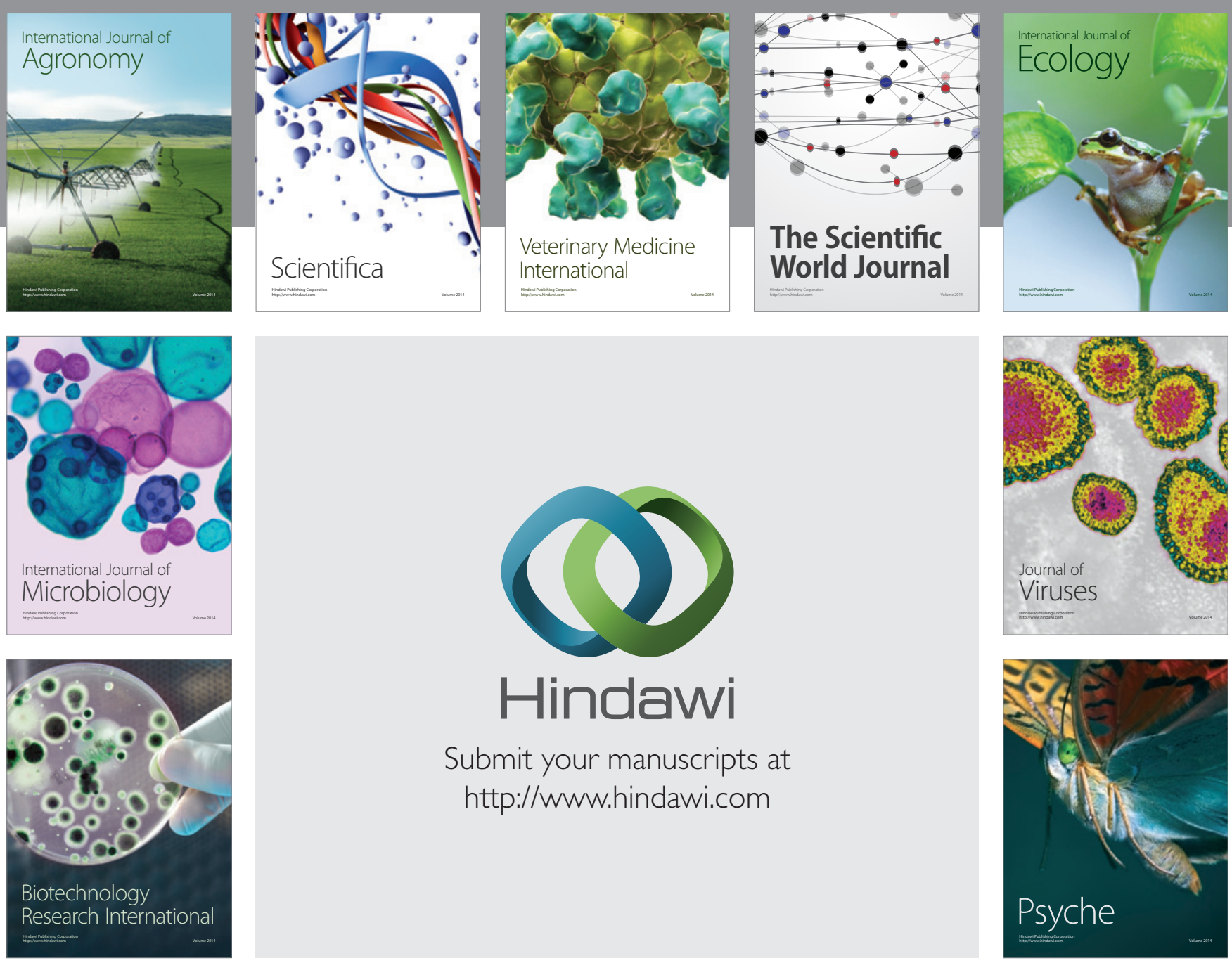

Submit your manuscripts at

http://www.hindawi.com
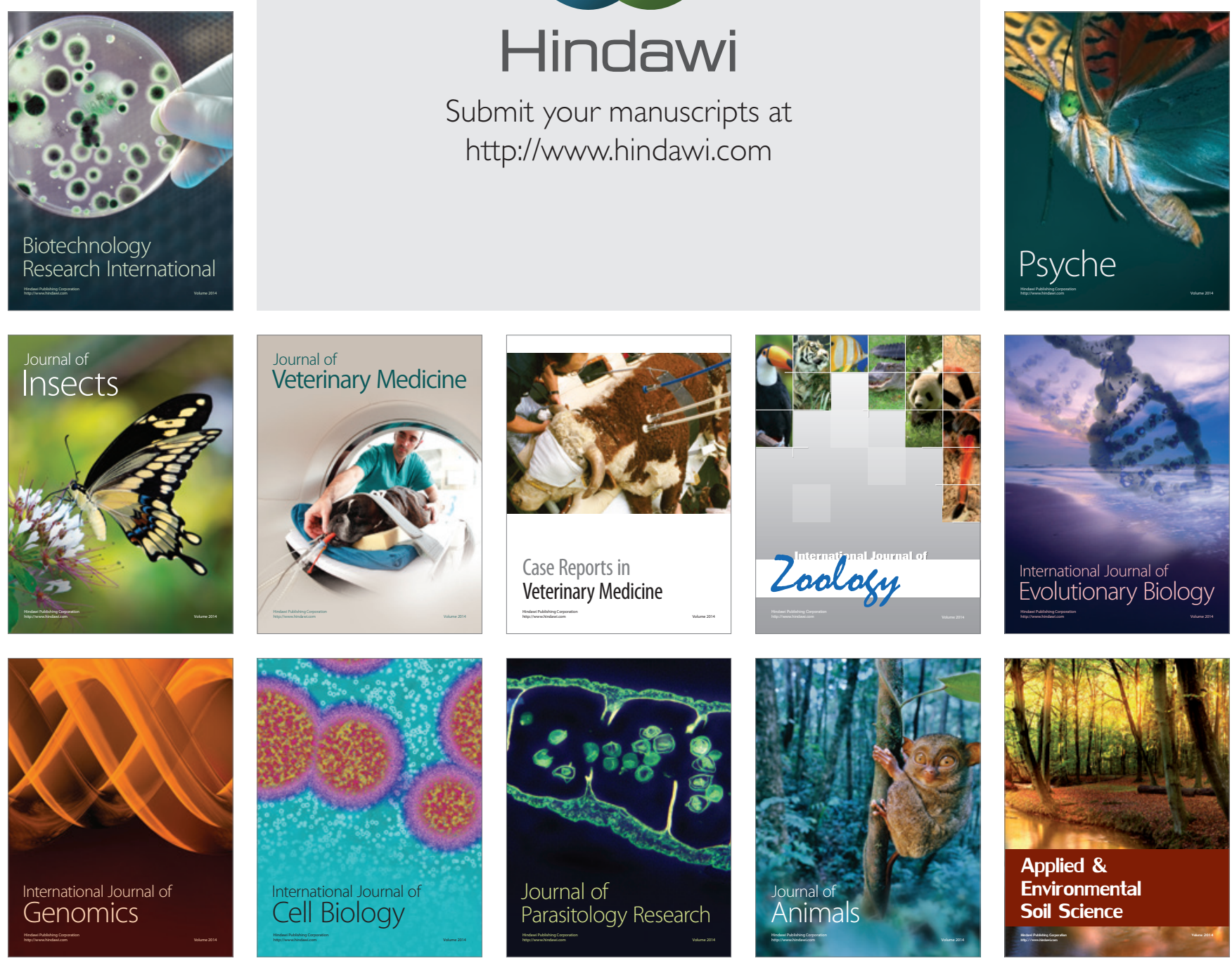